\title{
Direct numerical simulation of a low momentum round jet in channel crossflow
}

DOI:

10.1016/j.nucengdes.2016.12.018

\section{Document Version}

Accepted author manuscript

Link to publication record in Manchester Research Explorer

\section{Citation for published version (APA):}

Wu, Z., Laurence, D., \& Afgan, I. (2017). Direct numerical simulation of a low momentum round jet in channel crossflow. Nuclear Engineering and Design, 313, 273-284. https://doi.org/10.1016/j.nucengdes.2016.12.018

\section{Published in:}

Nuclear Engineering and Design

\section{Citing this paper}

Please note that where the full-text provided on Manchester Research Explorer is the Author Accepted Manuscript or Proof version this may differ from the final Published version. If citing, it is advised that you check and use the publisher's definitive version.

\section{General rights}

Copyright and moral rights for the publications made accessible in the Research Explorer are retained by the authors and/or other copyright owners and it is a condition of accessing publications that users recognise and abide by the legal requirements associated with these rights.

\section{Takedown policy}

If you believe that this document breaches copyright please refer to the University of Manchester's Takedown Procedures [http://man.ac.uk/04Y6Bo] or contact uml.scholarlycommunications@manchester.ac.uk providing relevant details, so we can investigate your claim.

\section{OPEN ACCESS}




\section{Direct Numerical Simulation of a Low}

2 Momentum Round Jet in Channel Crossflow

4 Zhao $\mathrm{Wu}^{1 *}$, Dominique Laurence ${ }^{1}$ and Imran Afgan ${ }^{1}$

$5{ }^{1}$ Modelling and Simulation Centre, School of Mechanical, Aerospace and Civil Engineering,

6 The University of Manchester, Oxford Road, Manchester M13 9PL,

7 zhao.wu@manchester.ac.uk

8 Abstract: Results of a direct numerical simulation of a jet in crossflow with passive scalar mixing are presented. The laminar jet issues from a circular exit into the channel crossflow with a low jet-to-crossflow velocity ratio of $1 / 6$. The governing equations are solved by Incompact3d, an open-source code combining the high-order compact scheme and Poisson spectral solver. An internal recycling approach is used to generate the fully turbulent channel flow profile. Four main flow structures are identified: 1) a large recirculation seen immediately downstream of the jet-exit; 2) a contour-rotating vortex pair formed from the stretching and reorientation of the injection-flow vorticity; 3) a horseshoe vortex generated as a result of the stretching of the vorticity at the jet-exit windward side; 4) shear layer vortices coming from the lifted and shed crossflow boundary layer vorticity. Passive scalar profiles show the mixing are strong in the shear layer where the crossflow fluid encounters the jet fluid. The database is made available online for public access.

\section{Introduction}

Jets in crossflow (JICF) are of great interests in wide ranges of industry applications. Examples can be found in turbine blades film cooling, de-icing of airplane wings, nuclear power-plant vessel emergency cooling or discharges into water or atmosphere. Numerous experiments (Andreopoulos \& Rodi 1984; Sherif \& Pletcher 1989; Fric \& Roshko 1994; Kelso \& Smits 1995; Kelso et al. 1996; Haven \& Kurosaka 1997; Smith \& Mungal 1998; Su \& Mungal 2004; Shan \& Dimotakis 2006), theoretically (Coelho \& Hunt 1989; Cortelezzi \& Karagozian 2001) and numerical studies (Yuan et al. 1999; Hoda \& Acharya 2000; Tyagi \& Acharya 2001; Muppidi \& Mahesh 2007; Sau \& Mahesh 2008) have been carried out on this problem, but most focused on cases with high jet to crossflow velocity ratio $R$. Industry applications with low $R$, such as wing de-icing and vessel cooling, have quite different flow features. However on fewer low $R$ cases have been studied, for example: Tyagi and 
1 Acharya's (2003) large eddy simulation on the film cooling, Duda's wing de-icing study 2 (2012) and Sau and Mahesh's theoretical study (2008) for $R=6$ to 1 only and jet Reynolds 3 number of 600.

4 In the jet in crossflow, the downstream development and passive scalar mixing is the result of 5 the momentum competition of the jet and the crossflow and an increasing influence of 6 turbulence in the far field downstream. Turbulence modelling is further complicated by near7 wall effects in the small velocity ratio $R$ case (Andreopoulos \& Rodi 1984). However, very

8 little data on turbulent quantities have been reported in the studies of low R cases.

9 Even fewer studies have examined scalar mixing, which is especially crucial for the low $R$ 10 applications. For example, in the wing de-icing system, the hot jet fluid will attach to the 11 wing to de-icy it; or in the vessel emergency cooling, the cold jet fluid will attach to the hot 12 pipe to prevent it from over-heating. Additional to this, little or no focus was given to the 13 temperature fluctuations on the wall surface or inside the solid wall, which can lead to severe 14 thermal stress fatigue particularly in the context of cooling injections in power plant safety 15 procedures.

16 Thus, the objective of present numerical simulation research programme is further understanding of the mean flow and turbulence characteristics of jets in crossflow at low velocity ratio and to obtain reliable and comprehensive turbulence data which can be used for testing and improving turbulence models. This numerical simulation research programme includes (a) direct numerical simulations (DNS) of JICF with passive scalar mixing, (b) validating the RANS models using the current database and (c) DNS of JICF with conjugate heat transfer. Part of the database is available online (http://dx.doi.org/10.17632/7nx4prgjzz.1) 23 at the time this paper is accepted (Wu, Laurence \& Afgan 2016). The testing and validating of RANS turbulent models using the whole database will be presented in another future paper. The results of DNS of JICF with conjugate heat transfer was submitted (Wu, Laurence, Iacovides, et al. 2016).

Section 2 describes the flow configuration. The DNS numerical techniques are provided in 28 Section 3, while its validation is discussed in Section 4. The general flow characteristics are described in some details in Section 5, followed by time-averaged profile analyses in Section 6 and instantaneous field discussions in Section 7. 


\section{Flow configuration}

2 Figure 1(a) shows the schematic view of the flow configuration of the jet in crossflow and the coordinate system. Hereafter, the subscript notation " $c$ " and " $j$ " represent the crossflow and the jet, respectively. The dimensions of the complete computational domain are $24 \mathrm{H}, 2 \mathrm{H}$ and $6 H$ in the $x, y$, and $z$ directions respectively, and the diameter of jet-exit $D$ equals to the total channel height $2 H$. The origin point $O$ is located at the centre of jet-exit at the bottom wall. The Reynolds number based on the crossflow bulk velocity $\overline{u_{c}}$ and half channel height $H$ is set at $R e=\overline{u_{c}} H / v=3333$, where $v$ is the fluid kinematic viscosity. The corresponding friction Reynolds number $R e_{\tau}$ is 208 .

The velocity ratio $R$ of the jet bulk velocity to the crossflow bulk velocity is set at $\overline{u_{j}} / \overline{u_{c}}=$ $1 / 6$, which leads to the Reynolds number based on the jet bulk velocity $\bar{u}_{\jmath}$ and jet-exit diameter $D$ as 1111 . Thus, the jet is considered as laminar, and a parabolic profile can safely be imposed. However, as Andreopoulos (1985) mentioned, the interaction between the jet and pipe affects the pipe flow up to two diameters upstream below the junction. The present case would be difficult to set-up experimentally (using a sophisticated variable thickness honeycomb perhaps) and is therefore a purely numerical experiment similar to several other numerical works (Salewski et al. 2008; Bagheri et al. 2009; Muldoon \& Acharya 2010; Grout et al. 2011; Ilak et al. 2012), though with higher $R$.

The Prandtl number $\operatorname{Pr}=v / \alpha$ is 0.7 in this study, where $\alpha$ is the fluid thermal diffusivity. The temperature is normalised by the temperature differences between the jet and the crossflow, i.e. $\theta=\left(T-T_{c}\right) /\left(T_{j}-T_{c}\right)$. As the fluid properties are constant, the flow is considered incompressible and the temperature a passive scalar. The DNS set-up and flow features for the adiabatic wall thermal boundary condition $(Q=0)$ only is presented herein. Iso-thermal $(T=0)$ and conjugate heat transfer are compared in subsequent papers $(\mathrm{Wu}$, Laurence, Iacovides, et al. 2016).

The computational domain is discretized with $513 \times 193 \times 256$ (25.3 million in total) grid points in the $\mathrm{x}, \mathrm{y}$, and $\mathrm{z}$ directions, respectively. The grid points are uniformly distributed in $\mathrm{x}$ and $\mathrm{z}$ directions, but stretched in y-direction such that the near wall region is well refined. The grid spacing in wall unit is $\Delta x^{+}=10, \Delta y^{+}=0.7 \sim 7$ and $\Delta z^{+}=5$. In such grid, the round jet-exit is pixeled (see Figure 1(b)). This open-source DNS code, Incompact3d, has been applied to many curved surfaces (round impinging jet (Dairay et al. 2015), leading edge curvature effects (Lamballais et al. 2010), flow past a cylinder (Pinto et al. 2011) to name a few). 
1 The time step is fixed at $6 \times 10^{-4} H / \overline{u_{c}}$. For present DNS, cold flow simulation was first run.

2 Once the flow became fully developed, thermal equations were then added. The initial

3 temperature was set to zero for the whole domain. The total simulation time after the adding

4 of thermal equations is $6300 \mathrm{H} / \overline{u_{c}}$, of which $360 \mathrm{H} / \overline{u_{c}}$ simulation time-span is spent for the

5 initial temperature transients. The time-averaging profiles was accumulated during the

6 remaining $5940 \mathrm{H} / \overline{u_{c}}$ simulation time (corresponding to 247.5 whole channel flow-through

7 time).

8 The DNS simulations used 4096 CPU cores on a BlueGene/Q HPC. It takes 0.134s wall-

9 clock time per time step for this 25.3-million-cell mesh.

\section{Numerical method}

11 The governing equations are the continuity equation, the Navier-Stokes (NS) equation and 12 the energy equation:

$$
\begin{gathered}
\nabla \cdot \mathbf{u}=0, \\
\frac{\partial \mathbf{u}}{\partial t}+\frac{1}{2}[\nabla(\mathbf{u} \cdot \mathbf{u})+(\mathbf{u} \cdot \nabla) \mathbf{u}]=-\nabla p+\frac{1}{\operatorname{Re}} \nabla^{2} \mathbf{u} \\
\frac{\partial \theta}{\partial t}+\nabla(\mathbf{u} \cdot \theta)=\frac{1}{\operatorname{RePr}} \nabla^{2} \theta
\end{gathered}
$$

13 where $\mathbf{u}$ is the dimensionless velocity vector, $t$ is the time, $p$ is the dimensionless pressure, 14 and $\theta$ is the dimensionless temperature. All variables are normalized by crossflow bulk 15 channel velocity $\overline{u_{c}}$ and channel half width $H$.

16 The DNS simulations are performed with Incompact3d; an open-source code developed 17 mainly by Laizet \& Lamballais and (2009) and Laizet \& Li (2011) from Université de 18 Poitiers and Imperial College London. All governing equations are solved on a collocated velocity grid via the six-order central compact finite different scheme (Lele 1992), while the pressure is on a staggered grid. A second-order Adams-Bashforth (AB) scheme is used for time-advancement and continuity is verified at the end of each sub-time step by solving a pressure Poisson equation. This Poisson equation is solved through a spectral solver to avoid the expensive cost of applying high-order scheme combined with iterative techniques. The solver is highly accurate and has been benchmarked on a wide range of cases and published in 40 papers (cf. http://www.incompact3d.com/), recently including heat transfer (Flageul et al. 2015). 


\section{$1 \quad 3.1$ Boundary conditions}

2 On both bottom and top walls, no-slip boundary conditions are imposed for the velocity field

3 and adiabatic condition for the temperature. The convective boundary condition (Ol'shanskii

4 \& Staroverov 2000) is applied to the outlet section. In current study with the spanwise size of

$53 D$, the periodic boundary condition is applied on the lateral directions to mimic a single row

6 of nozzles similar to that in (Tyagi \& Acharya 2003; Sakai et al. 2014). Majander and

7 Siikonen (2006) conducted a large eddy simulation with spanwise size of $8 D$ and free-slip

8 boundary conditions on the lateral surfaces. They obtained an agreement between their

9 simulation and the reference experimental data. The present narrower domain size will

10 restrict the spreading of the jet, or rather row of jets.

11 In order to generate fully turbulent channel flow before the jet-exit, an internal recycling 12 approach proposed by Lamballais (2014) is used. As illustrated in Figure 1, the velocity at the 13 cross section $x_{r}$ is copied to the inlet plane to be used as the Dirichlet inlet boundary 14 condition. This process can be mathematically expressed as $u^{n+1}(0)=u^{n}\left(x_{r}\right)$, where the 15 superscript denotes the time steps. To numerically achieve this only a short upstream length is required to ensure mimicking of a periodic channel flow. In present the study, the streamwise length of the recycling region is set to $10.59 H=3.37 \pi H$, which was found sufficient for performing DNS of a periodic channel flow. If the jet-exit was too close to the recycling region end, interactions of crossflow and jet at the windward side of jet-exit might "contaminate" the recycling region. Preliminary tests have shown that this upstream interaction only exists within $2 D=4 H$ upstream. So the jet-exit is safely set at $6.75 H$ further downstream of the end section of recycling region. This is confirmed by investigating the time-averaged statistics accumulated within the recycling region (see Section 4).

\section{Grid resolution and validation of recycling method}

Figure 2(a) and (b) shows examples of one-dimensional velocity spectra, in which $E_{i i}$ is the one-dimensional spectra, and $\kappa_{x}$ and $\kappa_{z}$ the streamwise and spanwise wavenumber respectively. Figure 2(a) shows the streamwise spectra at $z / H=0$ and $y / H=0.78$, and Figure 2(b) shows the spanwise spectra at $x / H=3.66$ and $y / H=0.78$. The $-5 / 3$ decay regions are seen in both figures, naturally limited at this low Reynolds number, after which sharply drop exists. The energy at high wavenumbers is several decades lower than that low wavenumbers. Figure 2(c) shows the compensated one-dimensional spanwise spectra 
$1 \quad \kappa^{5 / 3} E_{i i}$ at the center of channel, where the $E_{v v}\left(\kappa_{z}\right)$ and $E_{w w}\left(\kappa_{z}\right)$ are local isotropy at high 2 wavenumbers. Pope (2000) suggested a model spectrum for comparison:

$$
E(\kappa)=C \varepsilon^{2 / 3} \kappa^{-5 / 3} f_{\eta}(\kappa \eta)
$$

3 where $C \sim 1.5$ (Sreenivasan 1995) and $f_{\eta}(\kappa \eta)=\exp \left\{-\beta\left\{\left[(\kappa \eta)^{4}+c_{\eta}^{4}\right]^{1 / 4}-c_{\eta}\right\}\right\}$, if only 4 high wavenumbers are concerned. The present compensated spectra is in good agreement with Pope's model spectrum for Kolmogorov range, with $\beta=5.4$ and $c_{\eta}=0.41$, down to machine accuracy. Thus, it is concluded that the grid resolution is adequate for the current 7 configuration.

8 The inflow condition before the jet-exit is then validated (see Figure 3 and 4). Mean velocity, 9 Reynolds stresses and their budgets, averaged within the recycling region in spanwise and streamwise direction and time, are compared with reference data (Kasagi et al. 1992) obtained by spectral N-S equations solvers. Mean velocity, turbulent quantities and the budgets of Reynolds stresses are all in good agreement with reference data. The sum of budgets should theoretically be zero and imbalance measures unavoidable numerical errors (N-S equations discretization, post-processing manipulations, and incomplete statistical samples). Thus, the sum of the budgets can be regarded as a measure the accuracy of the results. The sum of the budgets in present simulations is at the same order of $1 \%$, as are reference DNS's. Indeed several independent researchers report 1.3\% maximum differences on the streamwise velocity and $7 \%$ on the rms profiles between different well established DNS channel flow data (Komen et al. 2014; Vreman \& Kuerten 2014). This indicates the present recycling technology with finite different scheme can generate reliable fully turbulent profiles at the same accuracy as reference periodic simulation which uses the spectral method. In present simulations, the sums of budgets are smoother that reference apart from oscillations very near the wall. This is due to the numerical scheme used in the code: homogeneous Neumann conditions are used in the pressure Poisson equation that leads to second-order accuracy at the border and cannot eliminate the Gibbs phenomenon there. In the range $10<y^{+}<30$ the peak of imbalance, which is not normalized, is simply because all terms are maximum in this buffer layer. Towards the central part of the channel, the imbalance is consistently smaller than the reference DNS.

Not only the low-order profiles but also the high-order ones are fit with reference's pure periodic channel flow statistics. The one-dimensional spectra fit well with Pope's spectrum 
1 model as discussed before (Figure 2(c)). These mean the recycling region is not affected by

2 the downstream jet, and clean fully turbulent inlet profiles are obtained before the jet-exit.

\section{Flow structures}

4 The flow of the jet in a crossflow is highly turbulent and complex. The time-averaged streamlines showing the main flow structures are presented in Figure 5.

6 When the crossflow meets the jet, they interact with each other; the jet is bent over by the 7 crossflow, while the crossflow is deflected upward and sideways by the jet. In the present case of the small $R$, the crossflow bends the jet immediately at the jet-exit, and the jet lifted the crossflow in turn. In high $R$ case, the jet would penetrate deeper into the crossflow, and thus the region immediately downstream of the jet would be similar to the near-wake behind a solid obstacle. However, in present low $R$ case, the jet is bent early, so the downstream field is more likely to be influenced by the turbulence from the jet. As a result, the wake region displays complex 3D flow patterns. The fluid near the symmetry plane is accelerated by the high-speed crossflow, and a transverse flow towards the symmetry plane is naturally induced because of the conservation of mass. A reverse-flow region exists downstream near the wall. The jet entrains the fluid at the lee side producing a low-pressure region there. This lowpressure region induces the fluid to enter this region laterally, and travel upstream due to the conservation of mass. This fluid is then entrained and elevated by the jet, and transported downstream.

As the upstream crossflow is lifted by the jet, the pipe flow fluid near the upstream part of exit is entrained into the region immediately upstream of jet-exit due to the requirement of the conservation of mass. A positive $\Omega_{z}$ vorticity is generated by this entrained fluid and stretched by $\partial w / \partial z$ at the immediately leading edge of jet-exit. The vortex lines are then bent around the jet and form a horseshoe vortex. This mechanism is quite different from that in JICF cases with higher $R$; in the high $R$ case, it is the oncoming crossflow reversed by the jet that forms the horseshoe vortex. The spanwise vortex converts to two streamwise vortices on the sides of symmetry plane, with negative value on the positive- $z$ side while positive value on the negative- $z$ side.

Besides the horseshoe vortex, another important feature in the vortex system of the jet in crossflow is the counter-rotating vortex pair. This vortex pair is located inside the two legs of horseshoe vortex and decays as it travels downstream. The parabolic-velocity-shaped jet 
1 introduces positive $\Omega_{x}$ in positive- $z$ and negative $\Omega_{x}$ in negative- $z$, and the vorticity is then carried downstream by the crossflow. As a result, the counter rotating vortices have opposite signs to the horseshoe vortex ones, i.e. positive $\Omega_{x}$ in positive- $z$ and negative $\Omega_{x}$ in negative- $z$. In this analysis, the counter-rotating vortex pair can be formed without computing the jet injection's upstream pipe, which is contrary to many previous researchers' ideas (Andreopoulos 1984, Coelho and Hunt 1989, Kelso, Lim and Perry 1996, Cortelezzi and Karagozian 2001), but coincides with Muppidi and Mahesh's (2006) conclusion. These authors concluded from their model that the pipe is not essential in generating the counterrotating vortex pair.

\section{Time-averaged results and discussion}

Several streamwise locations are chosen to present the time-averaged results obtained from present DNS (see Figure 6). Figure 7-12 show the mean velocity, temperature and temperature fluctuations developed along the $z$ planes, and how they vary with different distances calculated from the bottom wall where the jet issues. Two spanwise planes are chosen: the symmetry plane $(z / H=0)$ and the plane at the edge of jet-exit $(z / H=1)$.

\subsection{Mean velocities}

Figure 7 shows the downstream development of mean streamwise velocity at selected streamwise locations at $x / H$ between -1 and 5. On the symmetry plane (Figure 7(a)), a small negative velocity region is observed at the upstream edge of jet-exit. This is because the injection pipe fluid is entrained upstream as the crossflow is lifted by the jet. As explained in Section 5, the spanwise vortex formed by the reversed jet fluid is stretched by the accelerating flow circumventing the round jet and forms the horseshoe vortex. A wake behaviour is clearly seen from the velocity profile at downstream locations $(x / H=1.5,3$ and 5). At these locations, a large velocity gradient is obvious, indicating a free shear layer connecting the wake region with the accelerating crossflow due to the shallow channel height. At $x / H=1.5$ and 3 , reversed flow is clearly seen at the region very near to the bottom wall.

Arrows in last two profiles of Figure 7(a) highlight the near-wall acceleration along the side of the jet due to crossflow fluid sucked into the lower pressure wake region. This inflow is enhanced by the counter-rotating vortex pair. This inflow carries high-momentum fluid and causes this local maximum near the wall. This overshooting displays a wall-jet character, and is more pronounced in the cases of higher $R$ (Andreopoulos \& Rodi 1984). 
1 The streamwise velocity magnitude at plane $z / H=1$ (Figure $7(b)$ ) is larger than at $z / H=0$. 2 At $x / H=1.5$ and 3, the velocity increases as one approaches the bottom wall. The highspeed crossflow near the bottom wall is deviated by the jet and flows around the jet. High velocity gradient is not seen, implying the boundary of the jet does not spread to this plane. Further downstream at $x / H=5$ at both planes, the streamwise velocity profiles start to resume to a typical boundary layer shape. At this location, the velocity gradient $\partial\langle u\rangle / \partial y$ is much smaller which results a much lower turbulent production.

Figure 8 shows wall-normal velocity development as the flow goes downstream. At $z / H=0$, positive asymmetry velocity at $x / H=-1$ shows the jet lifts the crossflow over the full channel height. The same is seen for the profile at $x / H=1.5$ : positive velocity above the jetcrossflow shear layer $(y / H \sim 0.5$ referring Figure $7(a))$ also indicates the crossflow is lifted by the jet. The normal velocity changes sign at $x / H=3$ as result of the global downwash of crossflow as the near-wall separation bubble is now closing. Beneath the downwash of crossflow is an upward motion. This is due to the counter-rotating vortex pair bringing lateral fluid as described before. The counter-rotating vortex pair has positive $\Omega_{x}$ in positive- $z$ and negative $\Omega_{x}$ in negative- $z$, which also causes upward motion on the symmetry plane (refer to Figure 10(b)). The vortex cores of the counter rotating vortex pair is located at about $y / H=$ 0.2 at $x / H=1.5$, but move further away at downstream $(y / H=0.5$ at $x / H=3$ and $y / H=$ 0.6 at $x / H=5$ ). This behaviour can be seen from normal velocity profiles in which the $y-$ coordinate of maximum value move away from the bottom wall as one goes the downstream, and can be more easily identified from the vector plots in Figure 10.

Concluding on Figure 8, the wake region includes a downwash above the shear layer, and an upward motion under it, which together makes the normal profile with a positive value near the bottom wall and negative further away from the bottom wall. At $z / H=1$, the normal velocity is lower than at $z / H=0$. Positive normal velocity is seen at $x / H=-1,0$ and 1.5 indicating the crossflow is lifted by the jet as well. Large negative values are seen near the wall downstream as the counter-rotating vortex pair induces downward motion at the exitedge plane, which is opposite to the situation at the symmetry plane.

As the mean spanwise velocity is zero at the symmetry plane, only profiles at $z / H=1$ are shown (see Figure 9). It should be first noted negative spanwise velocity at $z / H=1$ plane presenting inflow towards the symmetry plane while positive spanwise velocity presenting the outward fluid motion. Positive spanwise velocity at $x / H=-1$ and 0 is observed due to 
1 the deflection of crossflow. The crossflow is diverted into two sides by the jet, thus the 2 positive value presenting this outflow motion is captured. The outward motion is quite strong, 3 the maximum value shown on Figure 9 is about $0.3 \overline{u_{c}} \sim 2 \overline{u_{j}}$. This results the spanwise 4 velocity gradient around $0.3 \overline{u_{c}} / H$ at this location. At downstream locations $(x / H=1.5$ and

53 ), negative values are shown near the wall due to the inward motion caused by the low 6 pressure after the jet. At $x / H=1.5$, the spanwise velocity changes sign as one goes away 7 from the bottom wall: inflow motion is seen near the bottom wall, while outflow motion is seen further away from the wall. This character implying a clockwise rotation still exists on positive- $z$, and this clockwise rotation is the foot of counter-rotating vortex pair (refer to Figure 10(a)). From Figure 10, we see that the counter-rotating vortex pair converges towards symmetry plane. The maximum negative spanwise velocity decreases as the x-coordinate increases, which might imply the counter-rotating vortex pair decays at downstream. The spanwise velocity increases near the wall, and even goes positive at $x / H=5$. This indicates the existence of another vortex pair with its sign opposite to the dominant one. Figure 10(c) points out this secondary vortex pair beneath the dominant one. Andreopoulos and Rodi (1984) also inferred the existence of this secondary pair from their coarser experimental results (first measure point was too far away from the wall).

\subsection{Mean temperature and its fluctuations}

The temperature has been non-dimensioned by the jet temperature and crossflow temperature, making $\theta=\left(T-T_{c}\right) /\left(T_{j}-T_{c}\right)$, where $T_{c}$ is the temperature of crossflow fluid while $T_{j}$ the jet fluid temperature. The thermal boundary conditions of both bottom and top walls are adiabatic, i.e. $\partial \theta /\left.\partial n\right|_{\text {wall }}=0$. Cases with iso-thermal condition $\left(\left.\theta\right|_{\text {wall }}=0\right)$ and fluid-solid conjugate heat transfer are discussed in a companion paper. Figure 11 shows the dimensionless temperature along the symmetry plane $(z / H=0)$ and $z / H=1$. On the symmetry plane, the temperature above the jet-exit is already partially mixed already at $x / H=0$, by large scale structures. In the downstream near field of $x / H=1.5$ and 3 , a maximum value is seen as cold crossflow is brought from the sides. This maximum temperature value decrease as one goes further away from jet-exit, but its wall normal position rises first then falls. The drop of the maximum value indicates the jet and the crossflow are further mixing and the trend of the position of this maximum value indicates the penetration and the downwash of jet in the downstream field. On the side, a local maximum is also seen at the wall at $x / H=1.5$ which is due to the steady side vortices discussed earlier. 
1 Figure 12 shows the temperature fluctuations. The peak value indicates the strongest mixing.

2 The peak rises as $x$ increases and matches the shear layer positions shown in Figure 7. This maximum value of temperature fluctuations decreases rapidly downstream. The temperature fluctuation values on the wall are of utmost importance for thermal fatigue studies. Nusselt number values for non-adiabatic cases and RANS predictions are shown in a subsequent paper.

\section{$7 \quad 7$ Instantaneous results and discussion}

8 Averaged profiles above have shown some important structures in the jet in crossflow, like 9 the horseshoe vortex, counter-rotating vortex pair and the recirculation after the jet. However as the flow has large-scale unsteadiness, some dynamic structures will be hidden if only the time-averaged profiles are examined.

\subsection{Shear layer vortices are hairpin vortices}

13 Figure 13 shows iso-surface of $\mathrm{Q}$-criterion $(Q=15)$ coloured by streamwise velocity at $t=t_{0}$.

14 The horseshoe vortex is evident. The recirculation is observed (see the negative streamwise velocity region at downstream in Figure 13). Several Hairpin-shaped vortex structures are seen as well.

Sau and Mahesh (2008) explain that the relative vorticity in the crossflow boundary layer increases as the velocity ratio decreases. Thus for $R=1.0$, the vorticity in the crossflow is almost the same as that in the upstream side of the jet-exit (but with opposite sign), so the vorticity at the windward side of the nozzle exit is cancelled. As a consequence, the vortex ring (shear layer vortices), which is often seen in the jet in crossflow with higher velocity ratio, due to the roll-up of the shear layer, does not form. So for $R=1$, in Sau and Mahesh, the flow structures are only made of by the rolling-up on the downstream side alone, and this rolling-up forms the hairpin vortex structures eventually.

The case is different in present simulation. The different signs of vorticity at the boundary layer of crossflow and windward side of jet-exit are also seen in present simulation. However, the velocity ratio here $(R=1 / 6 \sim 0.17)$ is much smaller than that of Sau and Mahesh (2008). As seen in Figure 14(a), the vorticity of the crossflow boundary layer (in blue) is much larger than that of jet boundary layer (in red). In our case, the channel flow vorticity $(\mathrm{d} u / \mathrm{d} y)$ largely exceeds that of the jet front $(\mathrm{d} v / \mathrm{d} x)$. The former is then lifted and starts shedding even without the piston stroke effect used in Sau and Mahesh. 
1 Figure 14 shows the process of a shedding of the vorticity of crossflow boundary layer (see dashed arrow in Figure 14). The crossflow fluid flows over the upstream side of jet-exit

3 (Figure 14(a)). The jet vorticity rotates and stretches the crossflow boundary layer vortex

4 (Figure 14(b)), and finally the latter one breaks down (Figure 14(c)).

5 After the crossflow boundary layer vortex falls off, it develops as one goes downstream (see 6 solid arrow in Figure 14(c)). The lifted and hashed crossflow boundary layer vortex flows 7 downstream driven by the jet and mainstream crossflow together. The vortex extends in the spanwise direction, and the wall normal velocity across the vortex is higher in the middle but lower at two sides. This then bends the vortex to arch shape at first. As the streamwise velocity in the shear layer is higher than that near the wall, the top of the arch-shape vortex is carried downstream faster than its legs (as seen the pointed vortex in the middle). When the vortex is over jet, the jet flow is relatively stable, so the vortex can keep its integrity; then vortex stretching due to mean velocity difference between the top and bottom further energises it; when the vortex travels further downstream, the flow becomes complex and turbulent. The reversed back-flow is entrained by jet (see Figure 14(b)), rises and collides with the hairpin vortex. So finally, the hairpin vortex is fractured.

\section{Conclusions}

The present paper depicts the JICF flow characteristics of the jet to cross flow at low velocity ratio $R$, which are quite different from the high $R$ cases. The data in this paper is available online (http://dx.doi.org/10.17632/7nx4prgjzz.1). In low $R$ cases, the crossflow is deflected and lifted by the jet fluid, the latter usually not observed in high $R$ cases. The jet is bent over by the crossflow immediately at the jet-exit. In the immediately downstream of the jet-exit a short recirculation bubble exists in the low pressure the wake region. The low pressure induces lateral fluid towards the symmetry plane. The inflow fluid comes with highmomentum fluid on the lateral sides and contributes to the overshoot of streamwise velocity profile on $z / H=1$ plane. A shear layer, which is set up above the wake region, is also observed. The shear layer sustains strong velocity gradient as the streamwise velocity increases above wake region to the value higher than inflow channel $\overline{u_{c}}$. With the recirculation bubble closing further downstream, the lifted crossflow displays a downwash and the velocity profiles rapidly recovers to typical turbulent channel flow type.

The JICF generates several coherent structures in the vortex system. The counter-rotating vortex pair (CVP) is the dominant vortex feature in JICF. It is a longitudinal vortex pair. The 
1 analysis shows the CVP results from the stretching and reorientation of the injection-flow 2 vorticity. Andreopoulos and Rodi (1984) suggests that this is the dominant reason in 3 developing the CVP in low $R$ cases, while in high $R$ cases it mainly comes from the shearing 4 of the orthogonal jet and crossflow. The CVP induces upward fluid motion on the symmetry 5 plane near the bottom wall. The CVP extends to the end of computational domain and 6 remains strong further downstream. The injection fluid at the jet-exit windward side is 7 entrained upstream, since the crossflow is lifted by the jet. The spanwise gradient $\partial\langle w\rangle / \partial z$ stretches the vorticity from the entrained injection fluid, and the stretched vorticity rounds the jet-exit and forms the horseshoe vortex. The horseshoe vortex has an opposite sign of vorticity to the CVP. Instantaneous vortex profiles show the shear layer vortex comes from the lifted and shed crossflow boundary layer vorticity, which is also different from high $R$ cases.

The passive scalar profiles presented show the jet and the crossflow are rapidly mixed by the large structures, with strongest mixing occurring in the shear layer region at the front and above the jet while rms scalar fluctuations below it are much smaller.

\section{Acknowledgement}

17 The authors acknowledge the use of ARCHER HPC allocated via UK EPSRC Turbulence 18 Consortium (EPSRC grant EP/L000261/1) and the use of BlueGene/Q supercomputer sponsored by EDF R\&D centre Chatou. The authors are grateful to Cedric Flageul for help in implementing heat transfer in the DNS code and Sofiane Benhamadouche for valuable discussions and advice.

\section{References}

Andreopoulos, J., 1985. On the structure of jets in a crossflow. Journal of Fluid Mechanics, 157, p.163.

Andreopoulos, J. \& Rodi, W., 1984. Experimental investigation of jets in a crossflow. Journal of Fluid Mechanics, 138, p.93.

Bagheri, S. et al., 2009. Global stability of a jet in crossflow. Journal of Fluid Mechanics, 624, p.33.

Coelho, S.L. V. \& Hunt, J.C.R., 1989. The dynamics of the near field of strong jets in crossflows. Journal of Fluid Mechanics, 200, p.95. 
1 Cortelezzi, L. \& Karagozian, a. R., 2001. On the formation of the counter-rotating vortex pair in transverse jets. Journal of Fluid Mechanics, 446, pp.347-373.

3 Dairay, T. et al., 2015. Direct numerical simulation of a turbulent jet impinging on a heated wall. Journal of Fluid Mechanics, 764(1), pp.362-394.

5 Duda, B.M., 2012. Étude et analyse numérique d'un jet chaud débouchant dans un écoulement transverse en utilisant des simulations aux échelles résolues. Toulouse, ISAE.

Flageul, C. et al., 2015. DNS of turbulent channel flow with conjugate heat transfer: Effect of

Fric, T.F. \& Roshko, A., 1994. Vortical structure in the wake of a transverse jet. Journal of Fluid Mechanics, 279, pp.1-47.

Grout, R.W. et al., 2011. Direct numerical simulation of flame stabilization downstream of a transverse fuel jet in cross-flow. Proceedings of the Combustion Institute, 33(1), pp.1629-1637.

Haven, B.A. \& Kurosaka, M., 1997. Kidney and anti-kidney vortices in crossflow jets. Journal of Fluid Mechanics, 352, pp.27-64.

Hoda, A. \& Acharya, S., 2000. Predictions of a Film Coolant Jet in Crossflow With Different Turbulence Models. Journal of Turbomachinery, 122(3), p.558.

Ilak, M. et al., 2012. Bifurcation and stability analysis of a jet in cross-flow: onset of global instability at a low velocity ratio. Journal of Fluid Mechanics, 696, pp.94-121.

Kasagi, N., Tomita, Y. \& Kuroda, A., 1992. Direct Numerical Simulation of Passive Scalar

Kelso, R.M., Lim, T.T. \& Perry, A.E., 1996. An experimental study of round jets in crossflow. Journal of Fluid Mechanics, 306, p.111.

Kelso, R.M. \& Smits, A.J., 1995. Horseshoe vortex systems resulting from the interaction between a laminar boundary layer and a transverse jet. Physics of Fluids, 7(1), pp.153158.

Komen, E. et al., 2014. Quasi-DNS capabilities of OpenFOAM for different mesh types. 
Laizet, S. \& Lamballais, E., 2009. High-order compact schemes for incompressible flows: A simple and efficient method with quasi-spectral accuracy. Journal of Computational Physics, 228(16), pp.5989-6015.

Laizet, S. \& Li, N., 2011. Incompact3d: A powerful tool to tackle turbulence problems with up to $\mathrm{O}(105)$ computational cores. International Journal for Numerical Methods in Fluids, 67(11), pp.1735-1757.

Lamballais, E., 2014. Direct numerical simulation of a turbulent flow in a rotating channel with a sudden expansion. Journal of Fluid Mechanics, 745, pp.92-131.

Lamballais, E., Silvestrini, J. \& Laizet, S., 2010. Direct numerical simulation of flow separation behind a rounded leading edge: Study of curvature effects. International Journal of Heat and Fluid Flow, 31(3), pp.295-306.

Lele, S.K., 1992. Compact finite difference schemes with spectral-like resolution. Journal of Computational Physics, 103(1), pp.16-42.

Majander, P. \& Siikonen, T., 2006. Large-eddy simulation of a round jet in a cross-flow. International Journal of Heat and Fluid Flow, 27(3), pp.402-415.

Muldoon, F. \& Acharya, S., 2010. Direct Numerical Simulation of pulsed jets-in-crossflow. Computers \& Fluids, 39(10), pp.1745-1773.

Muppidi, S. \& Mahesh, K., 2007. Direct numerical simulation of round turbulent jets in crossflow. Journal of Fluid Mechanics, 574, pp.59-84.

Ol'shanskii, M.A. \& Staroverov, V.M., 2000. On simulation of outflow boundary conditions in finite difference calculations for incompressible fluid. International Journal for Numerical Methods in Fluids, 33(4), pp.499-534.

Pinto, L.C., Schettini, E.B.C. \& Silvestrini, J.H., 2011. Numerical analysis of the immersed boundary method applied to the flow around a forced oscillating cylinder. Journal of Physics: Conference Series, 296(1), p.12011.

Pope, S.B., 2000. Turbulent Flows, Cambridge: Cambridge University Press.

Sakai, E., Takahashi, T. \& Watanabe, H., 2014. Large-eddy simulation of an inclined round jet issuing into a crossflow. International Journal of Heat and Mass Transfer, 69, 
pp.300-311.

Salewski, M., Stankovic, D. \& Fuchs, L., 2008. Mixing in circular and non-circular jets in crossflow. Flow, Turbulence and Combustion, 80(2), pp.255-283.

Sau, R. \& Mahesh, K., 2008. Dynamics and mixing of vortex rings in crossflow. Journal of Fluid Mechanics, 604, pp.389-409.

Shan, J.W. \& Dimotakis, P.E., 2006. Reynolds-number effects and anisotropy in transversejet mixing. Journal of Fluid Mechanics, 566, pp.47-96.

Sherif, S.A. \& Pletcher, R.H., 1989. Measurements of the Flow and Turbulence Characteristics of Round Jets in Crossflow. Journal of Fluids Engineering, 111(2), pp.165-171.

Smith, S.H. \& Mungal, M.G., 1998. Mixing, structure and scaling of the jet in crossflow. Journal of Fluid Mechanics, 357, pp.83-122.

Sreenivasan, K.R., 1995. On the universality of the Kolmogorov constant. Physics of Fluids, $7(11)$, p. 2778.

Su, L.K. \& Mungal, M.G., 2004. Simultaneous measurements of scalar and velocity field evolution in turbulent crossflowing jets. Journal of Fluid Mechanics, 513, pp.1-45.

Tyagi, M. \& Acharya, S., 2001. Flow and Heat Transfer Predictions for Film-Cooling Flows Using Large Eddy Simulations,

Tyagi, M. \& Acharya, S., 2003. Large Eddy Simulation of Film Cooling Flow From an Inclined Cylindrical Jet. Journal of Turbomachinery, 125(4), p.734.

Vreman, A.W. \& Kuerten, J.G.M., 2014. Comparison of direct numerical simulation databases of turbulent channel flow at Re $\tau=180$. Physics of Fluids, 26(1), p.15102.

Wu, Z., Laurence, D., Iacovides, H., et al., 2016. Direct Simulation of Conjugate Heat Transfer of Jet in Channel Crossflow (submitted). International Journal of Heat and Mass Transfer.

Wu, Z., Laurence, D. \& Afgan, I., 2016. Direct simulation of hot jet in cold channel crossflow with adiabatic thermal boundary conditions. Mendeley Data, vl.

Yuan, L.L., Street, R.L. \& Ferziger, J.H., 1999. Large-eddy simulations of a round jet in crossflow. Journal of Fluid Mechanics, 379, pp.71-104. 


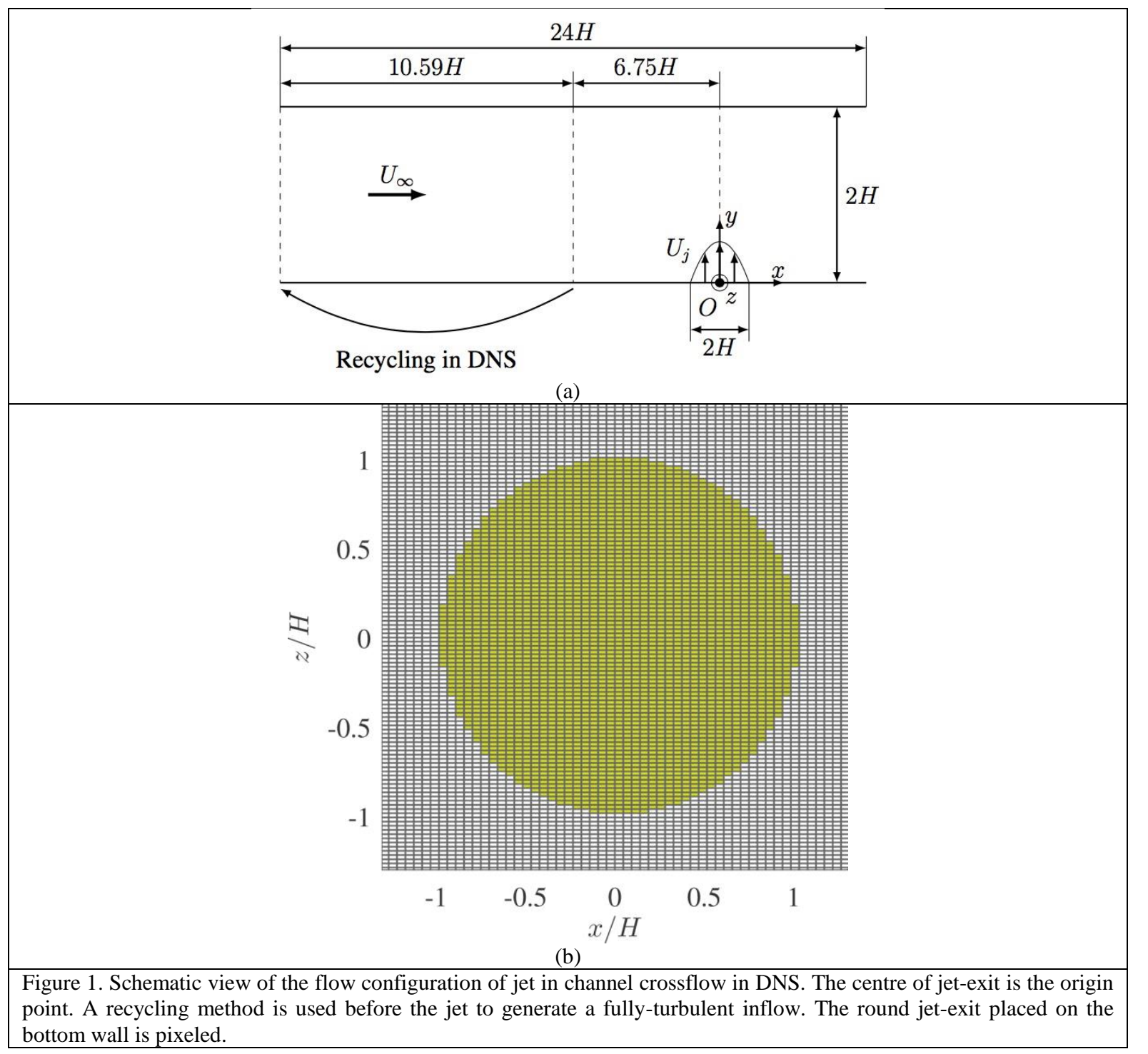

1

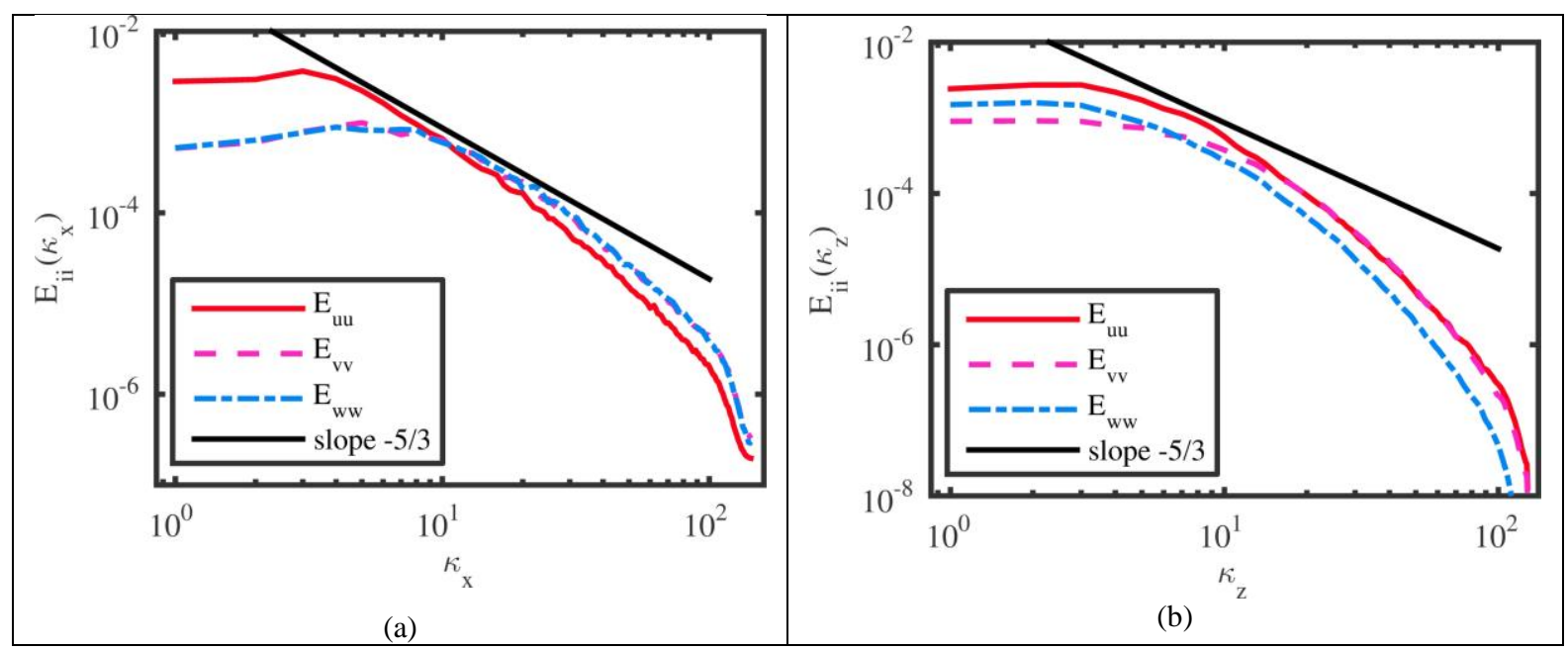




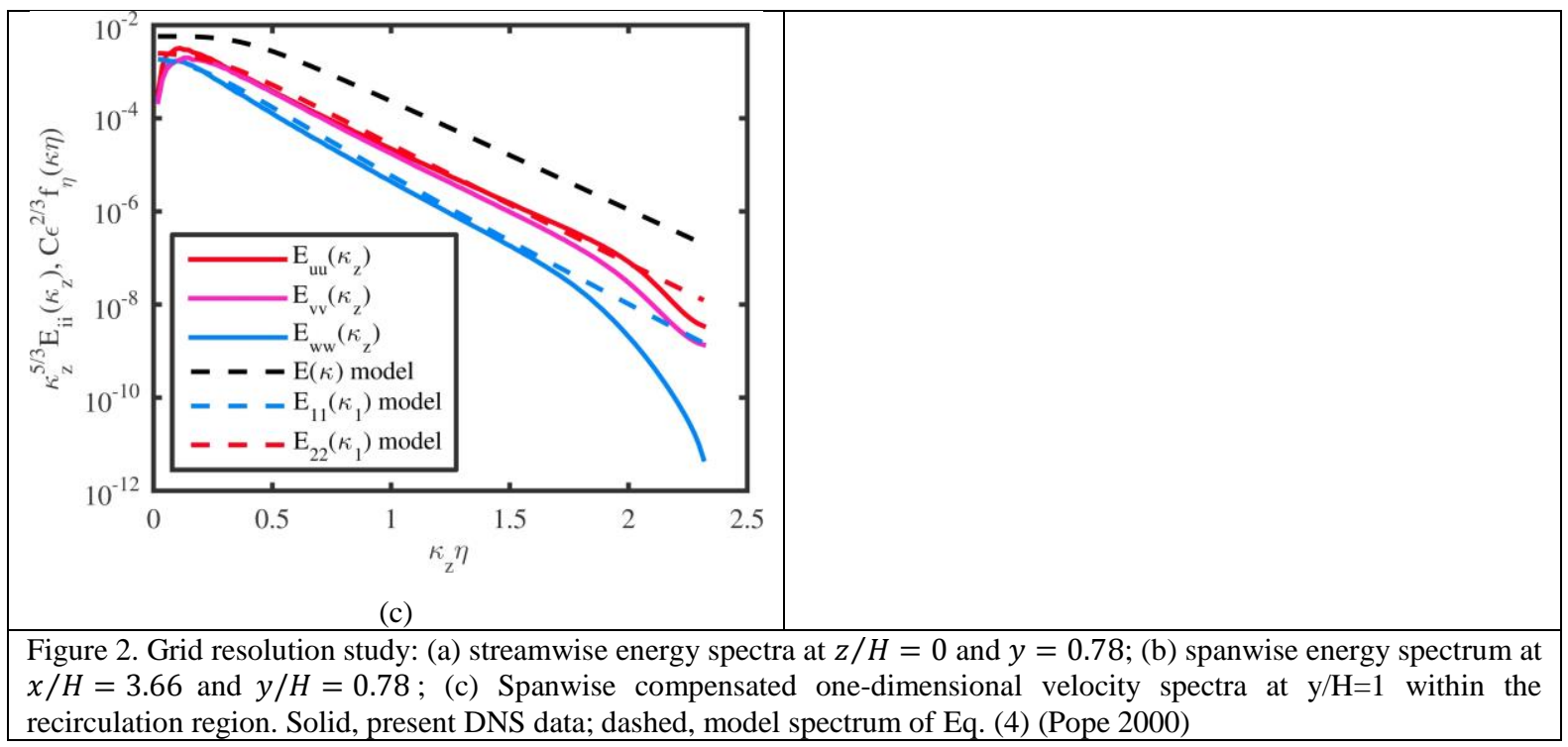

1

2 

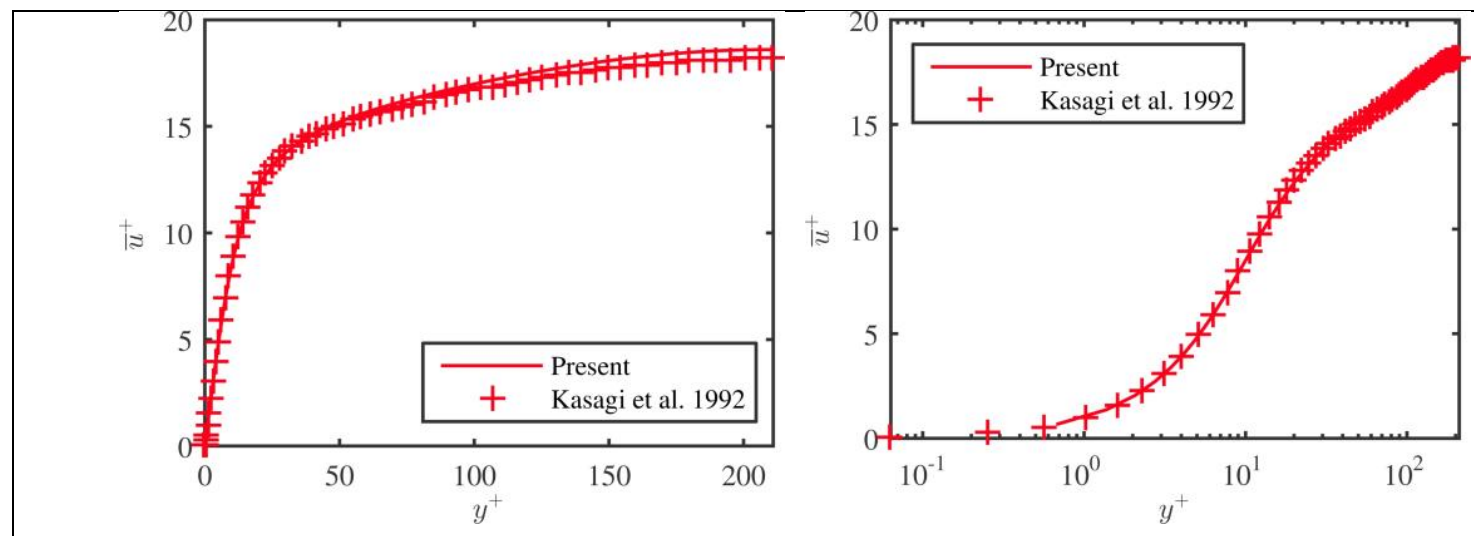

(a)
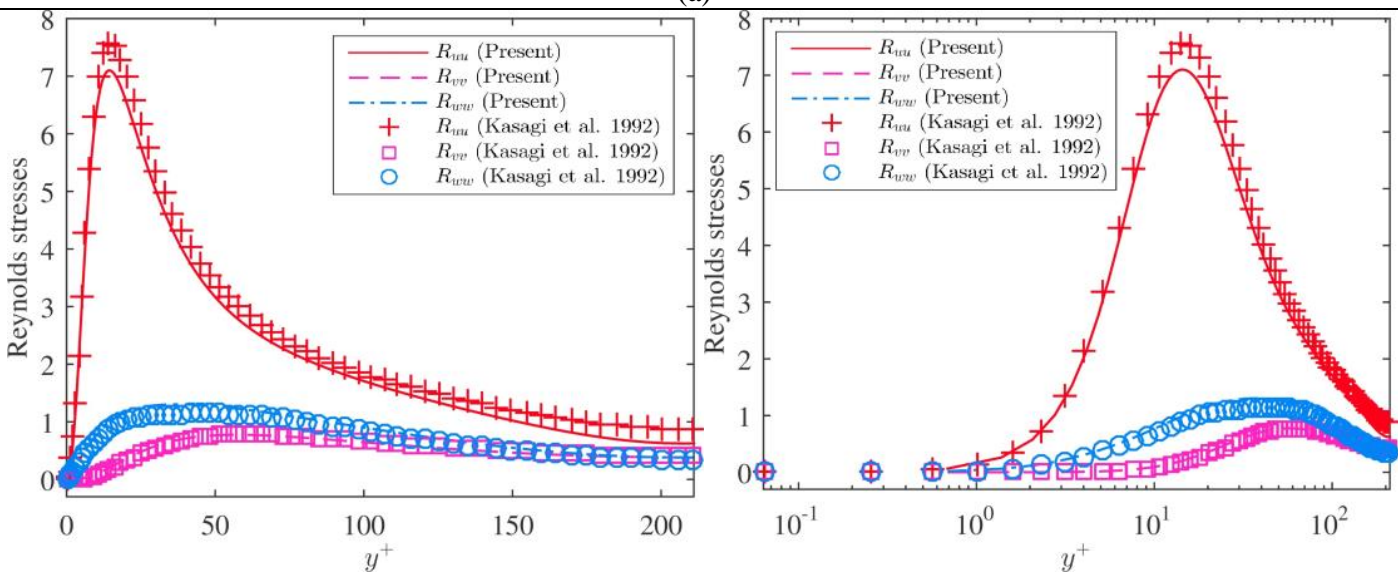

(b)

Figure 3. Non-dimensioned mean (a) streamwise velocity and (b) Reynolds stresses. Symbol, (Kasagi et al. 1992); line, present recycling results. 

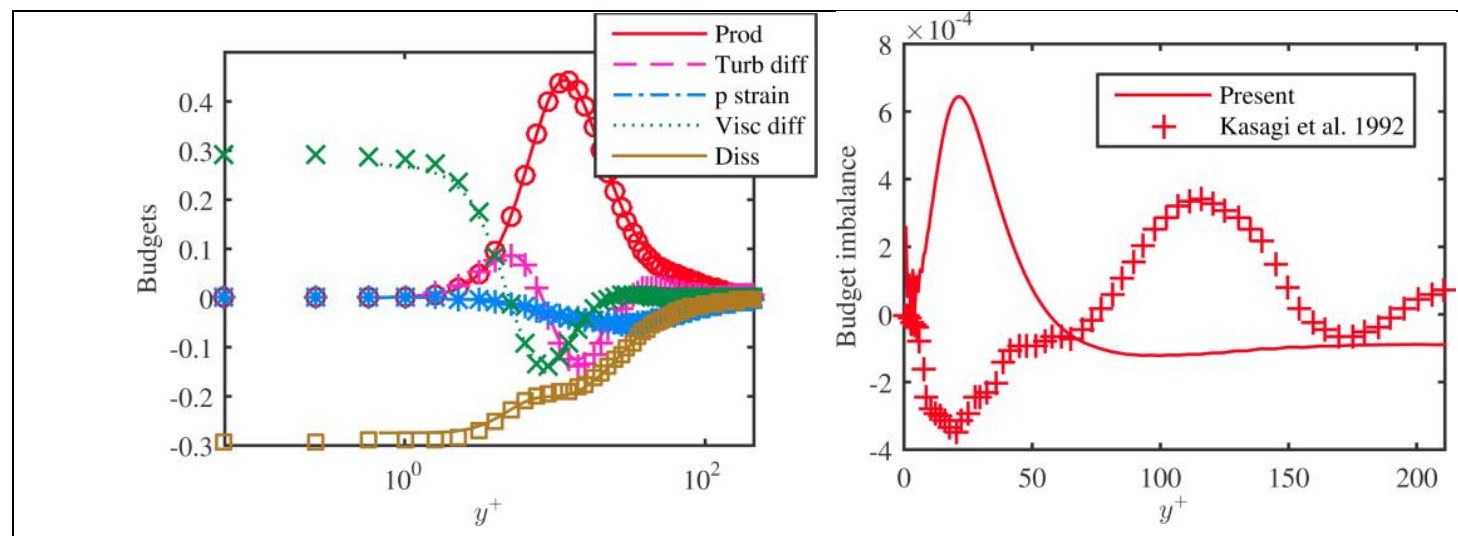

(b) $R_{u u}$ budget and imbalance
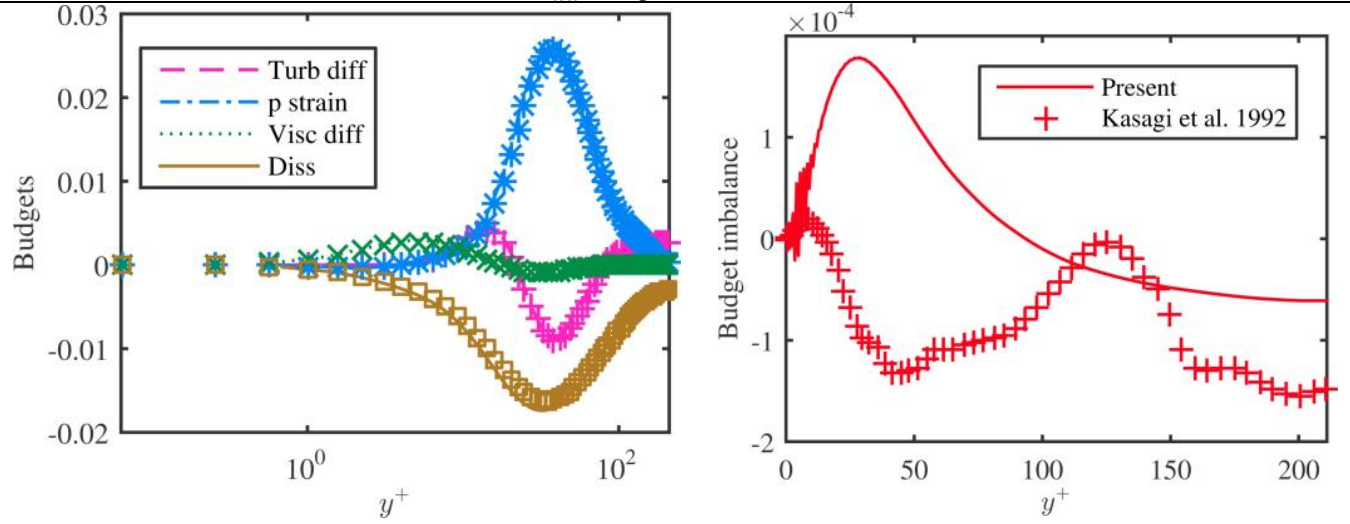

(d) $R_{v v}$ budget and imbalance
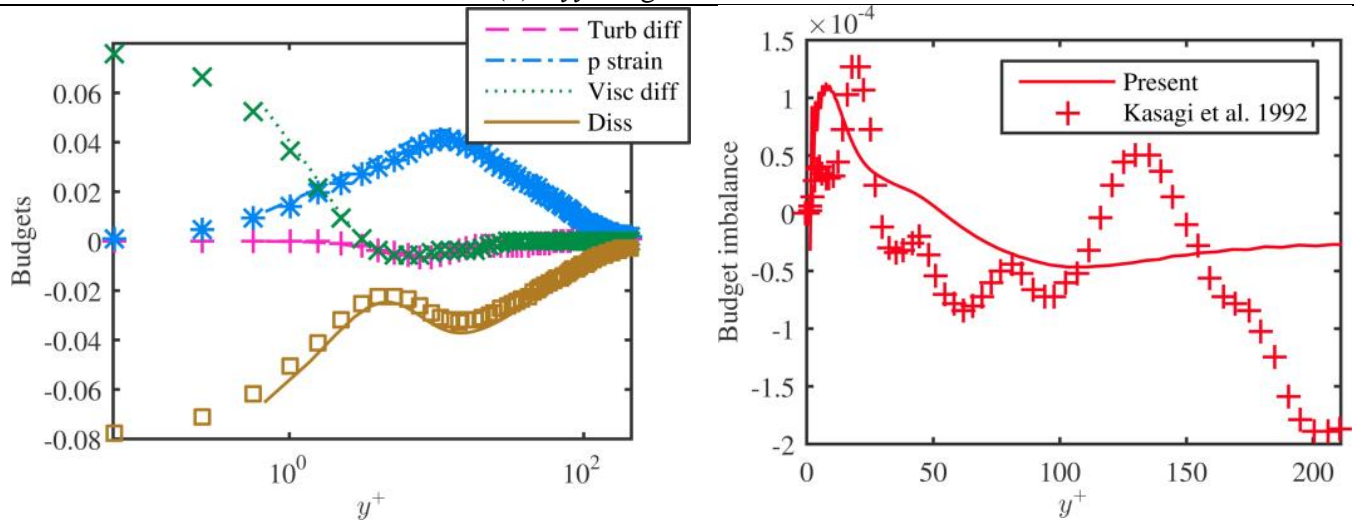

(f) $R_{w w}$ budget and imbalance
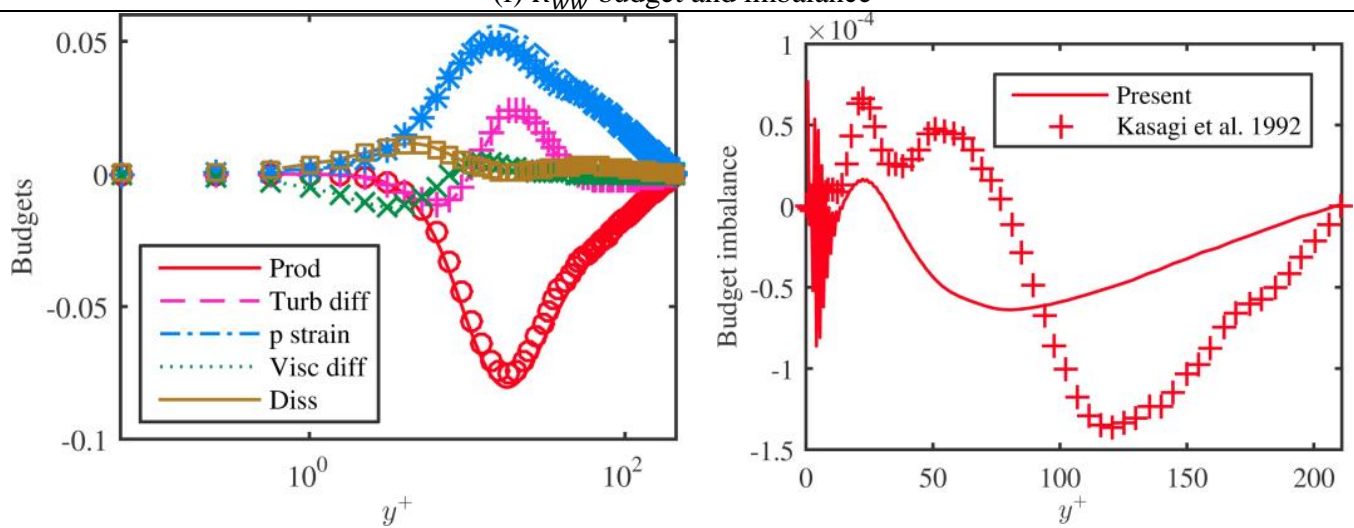

(h) $R_{u v}$ budget and imbalance

Figure 4. Budgets of Reynolds stresses (left) and their sum (right). From top to bottom: $R_{u u}, R_{v v}, R_{w w}$ and $R_{u v}$. Symbol, (Kasagi et al. 1992); line, present recycling results. 


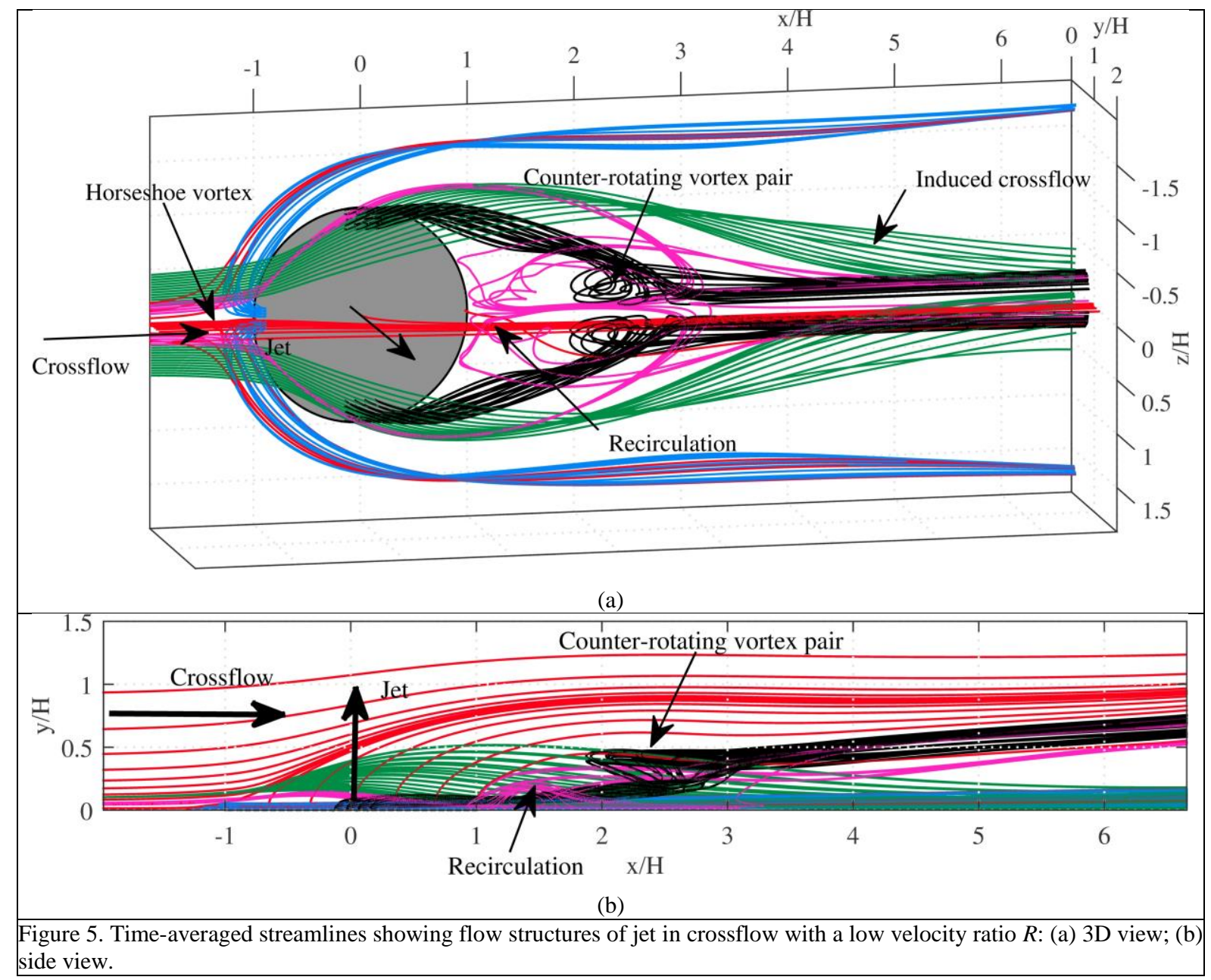

1

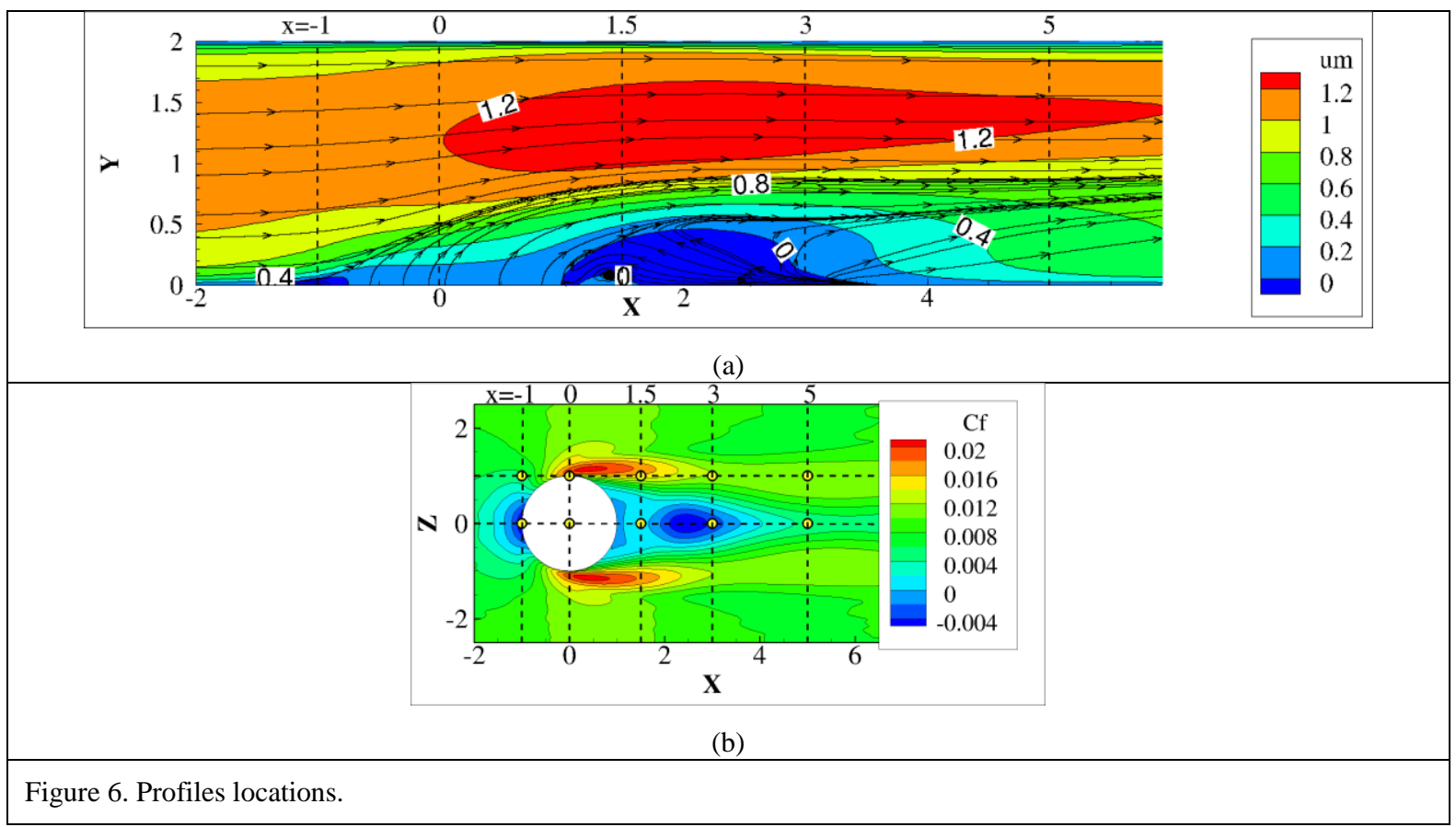



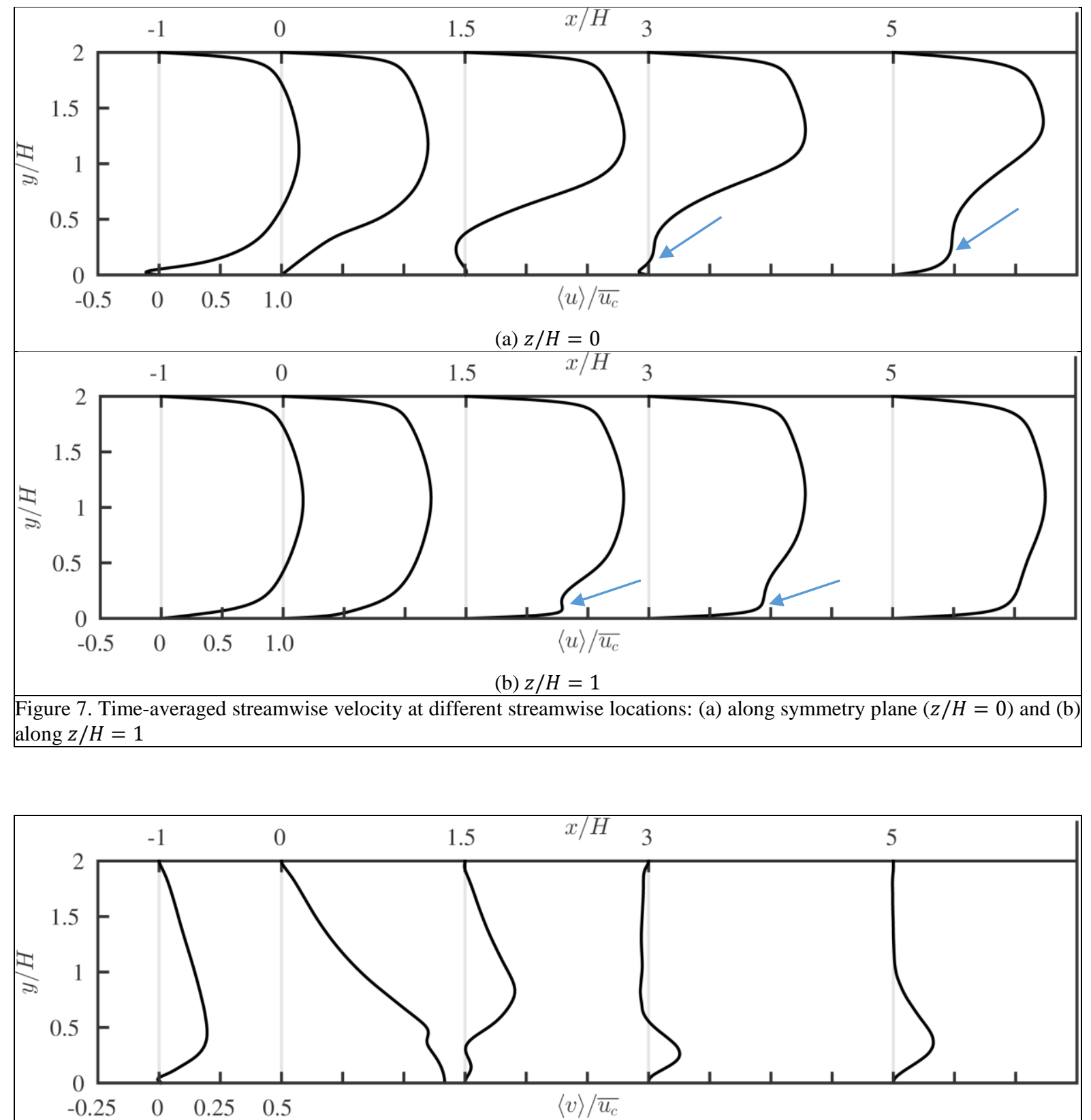

(a) $z / H=0$

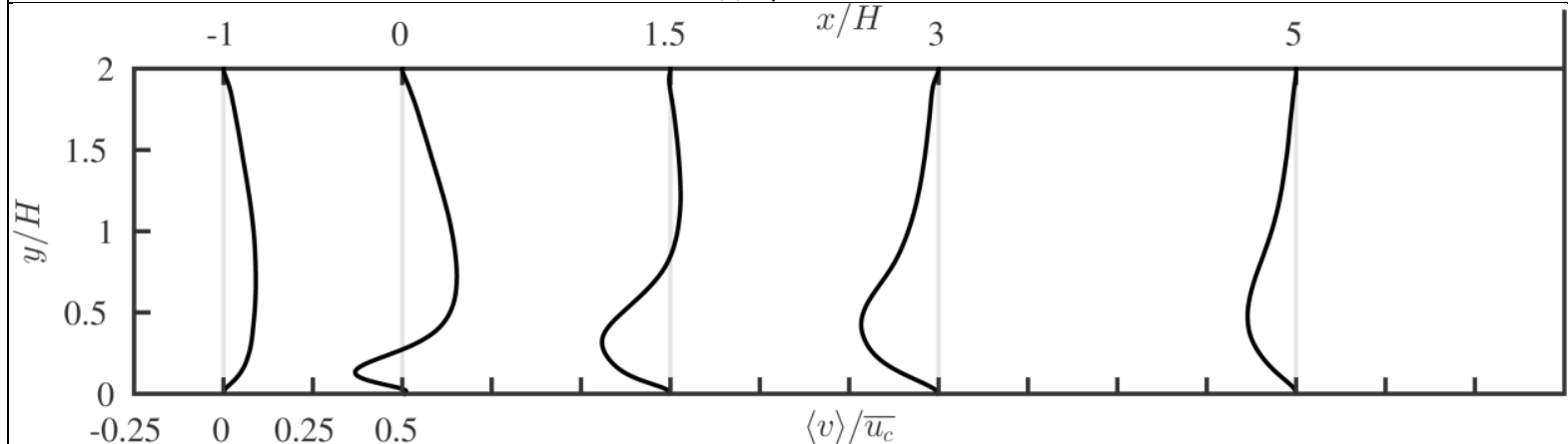

(b) $z / H=1$

Figure 8. Time-averaged normal velocity at different streamwise locations: (a) along symmetry plane $(z / H=0)$ and (b) along $z / H=1$ 


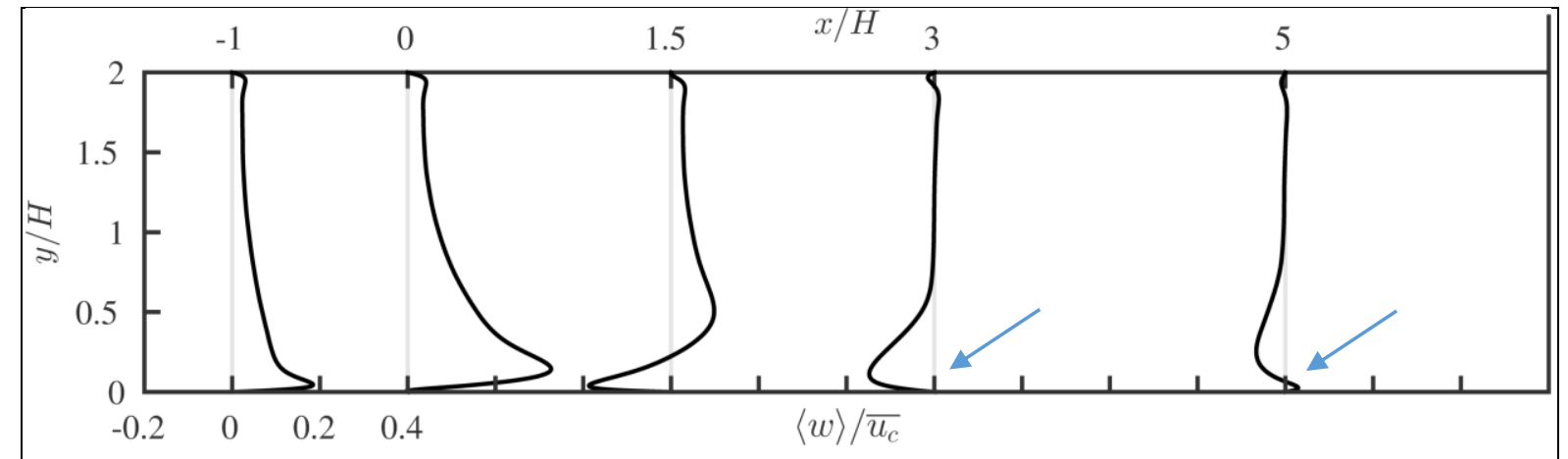

Figure 9. Time-averaged spanwise velocity at different streamwise locations along $z / H=1$

1

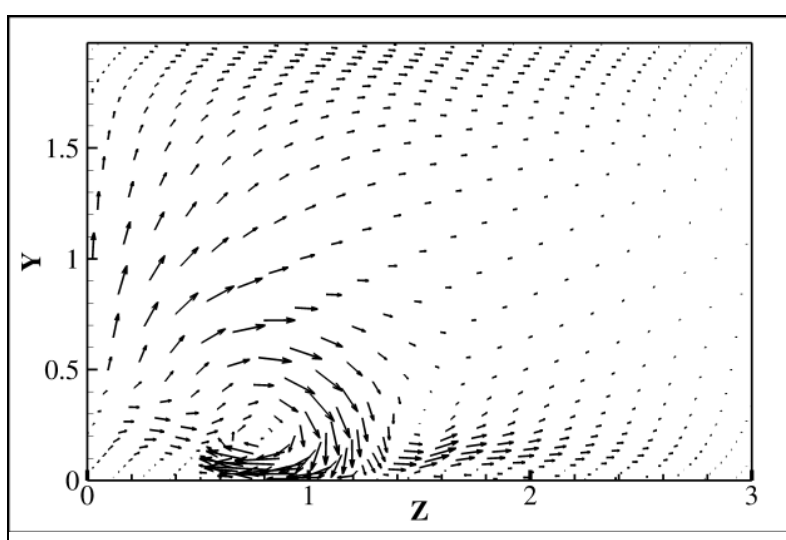

(a) $x / H=1.5$

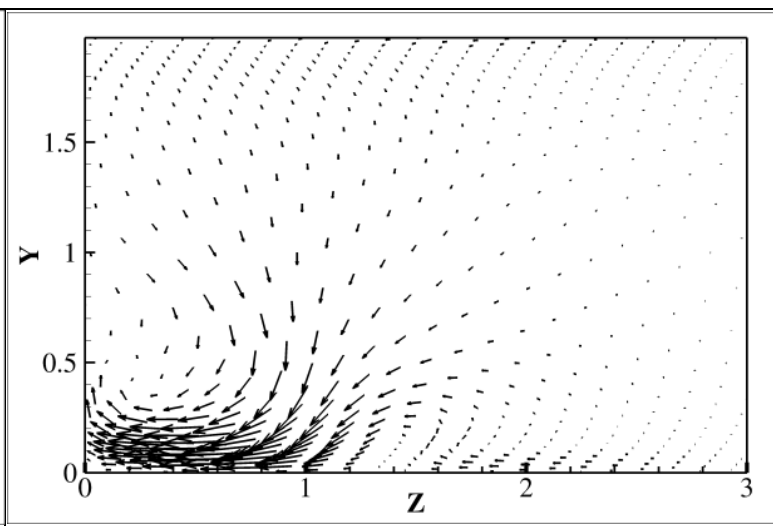

(b) $x / H=3$

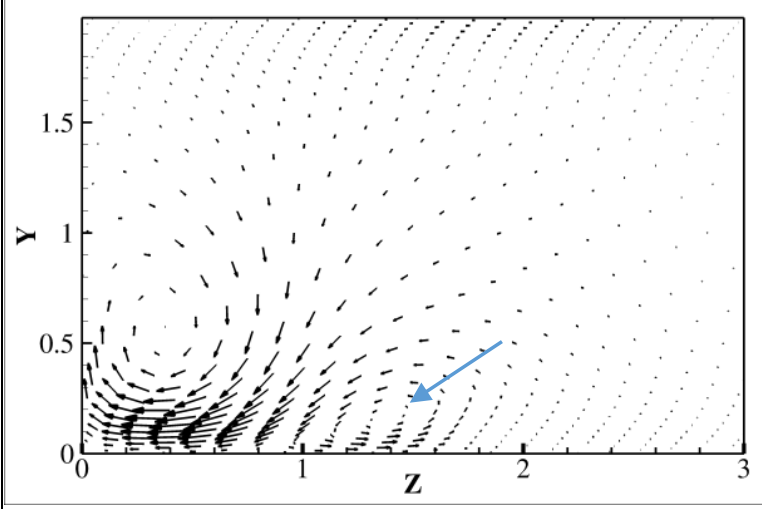

(c) $z / H=5$

Figure 10 . Velocity vectors at plane $x / H=1.5, x / H=3$ and $x / H=5$ 


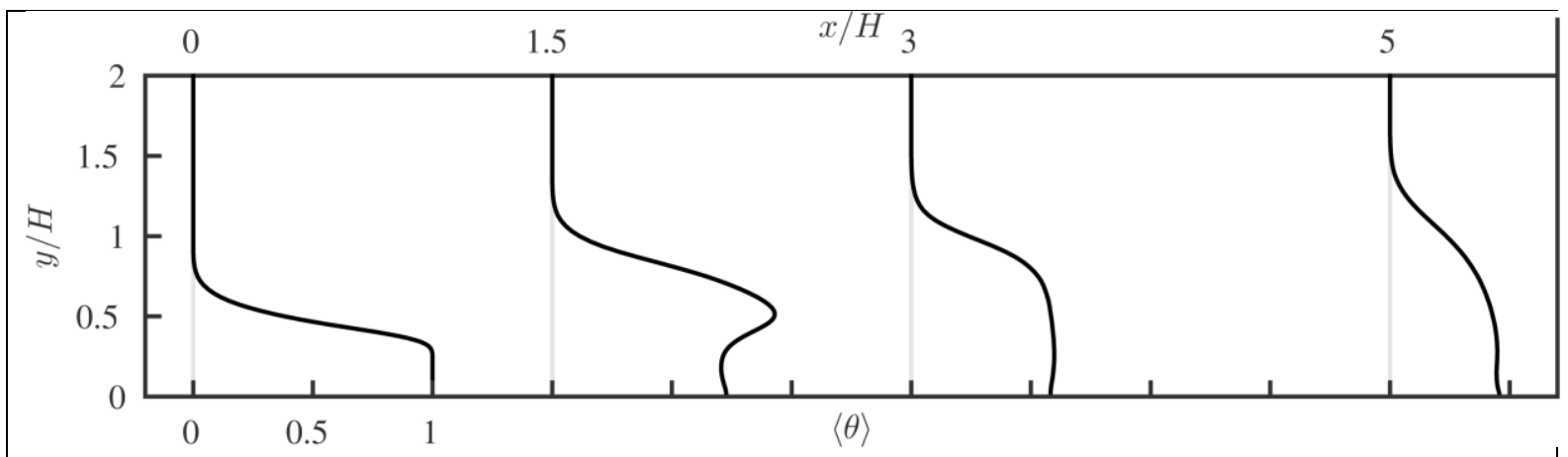

(a) $z / H=0$

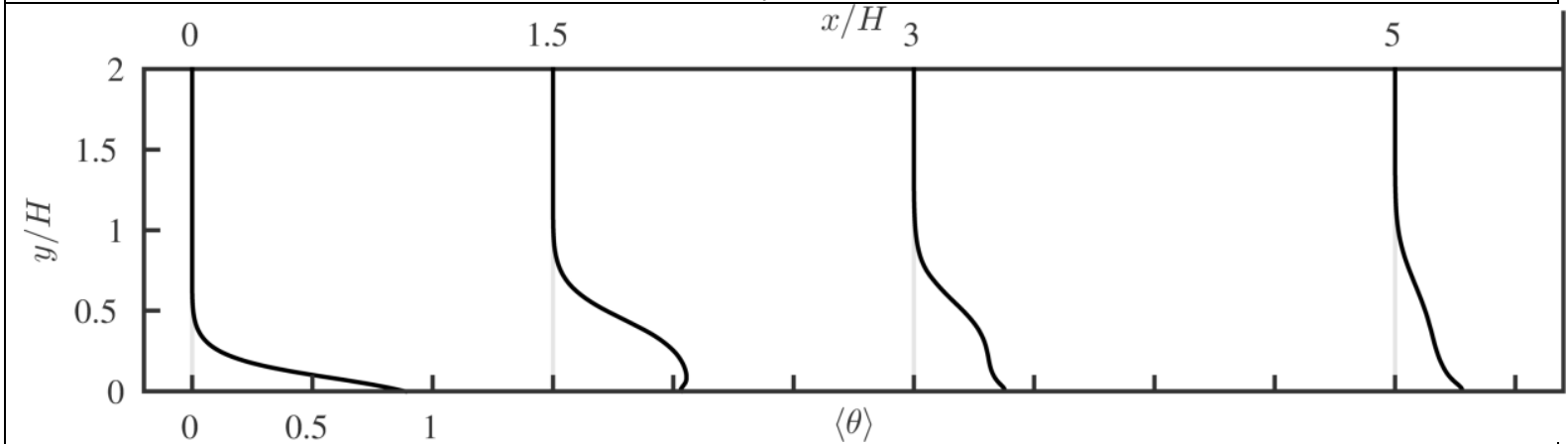

(b) $z / H=1$

Figure 11. Time-averaged dimensionless temperature $\langle\theta\rangle$ at different streamwise locations: (a) along symmetry plane $(z / H=0)$ and (b) along $z / H=1$

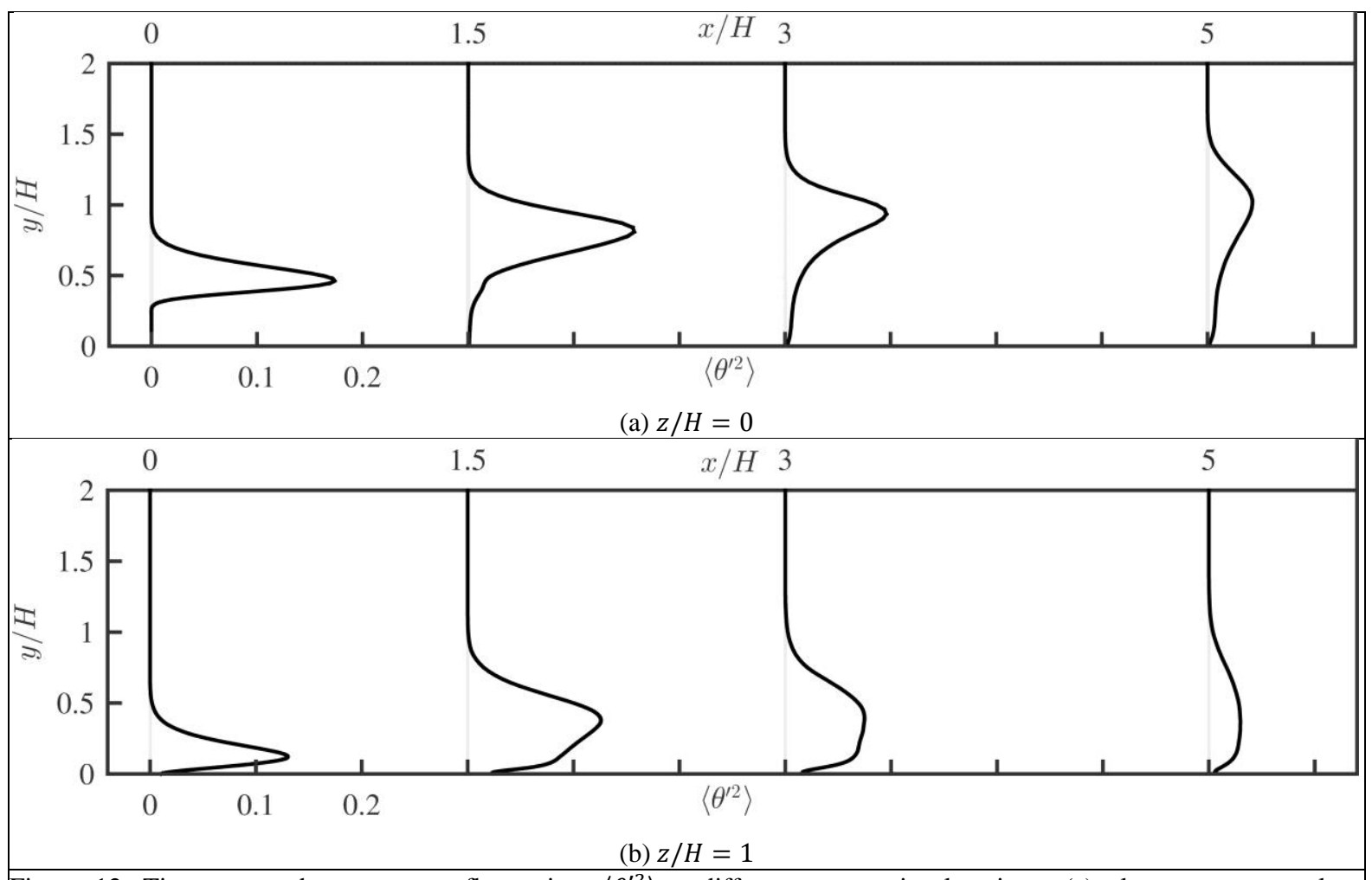

Figure 12. Time-averaged temperature fluctuations $\left\langle\theta^{\prime 2}\right\rangle$ at different streamwise locations: (a) along symmetry plane $(z / H=0)$ and (b) along $z / H=1$ 


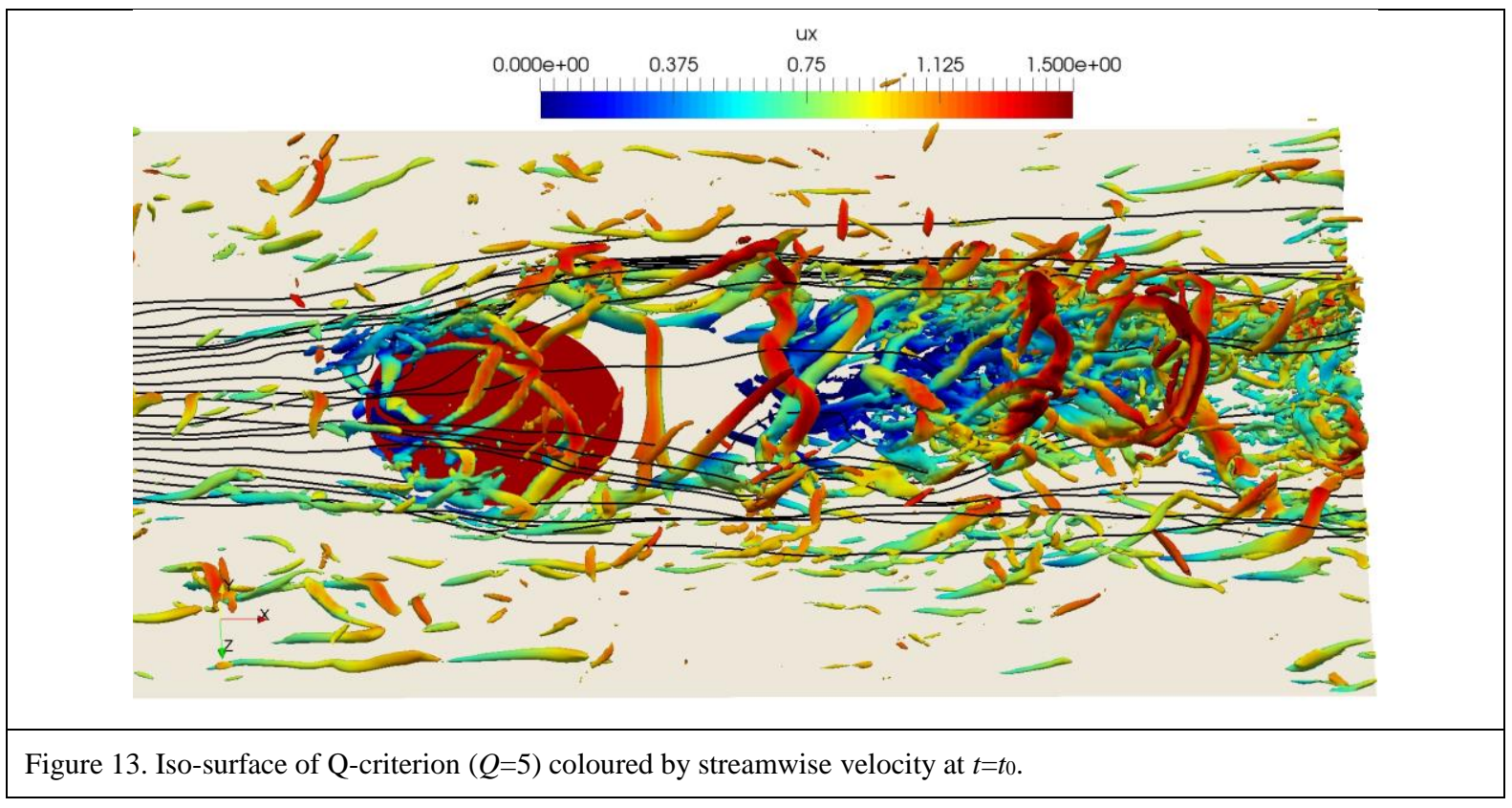




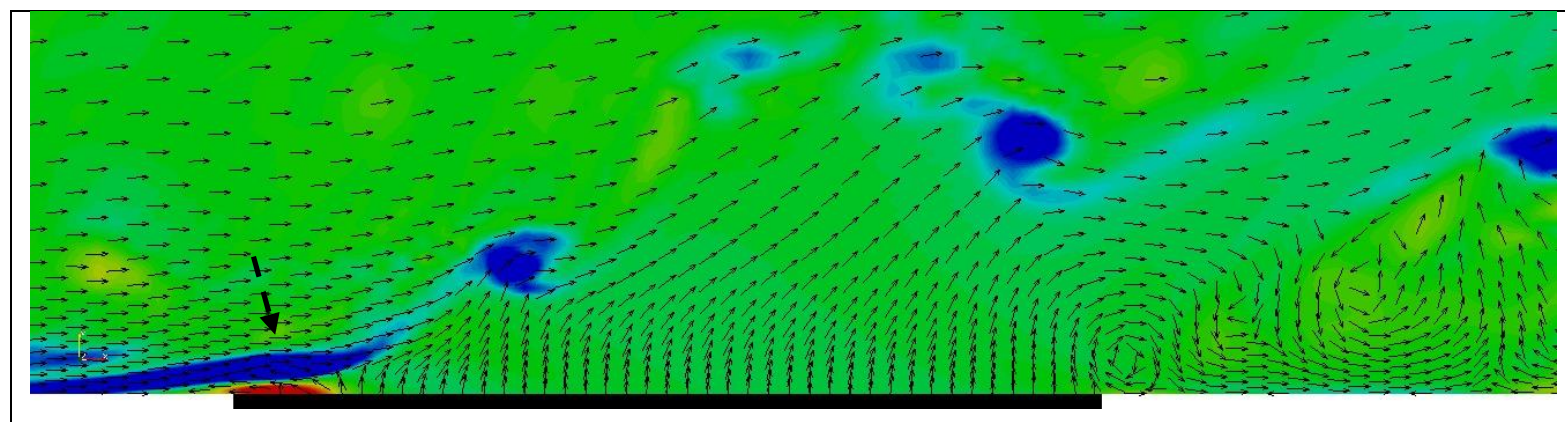

(a) $t=t_{0}+2.40$

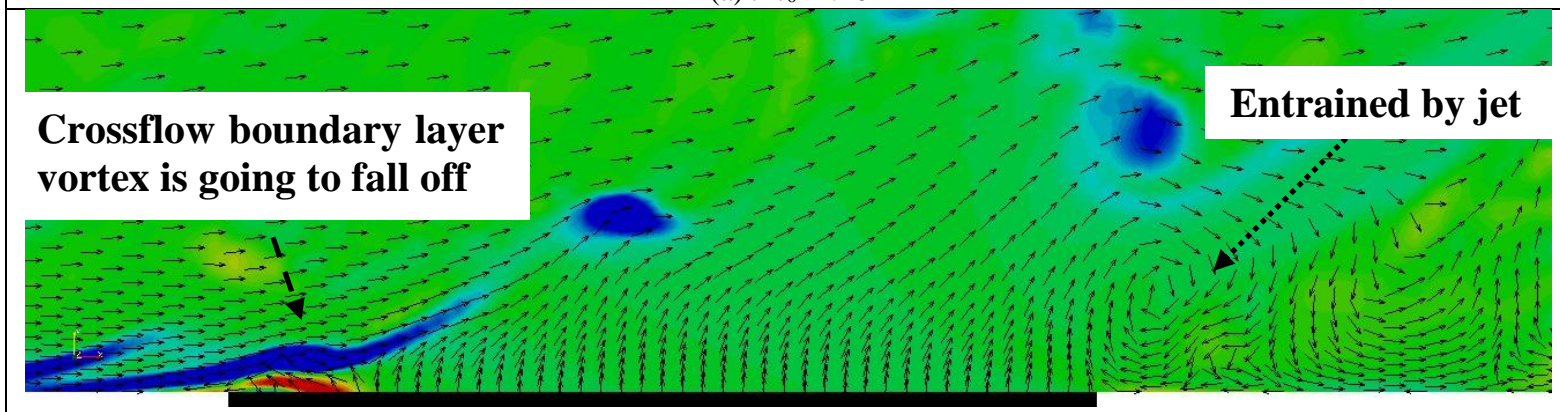

(b) $t=t_{0}+2.76$
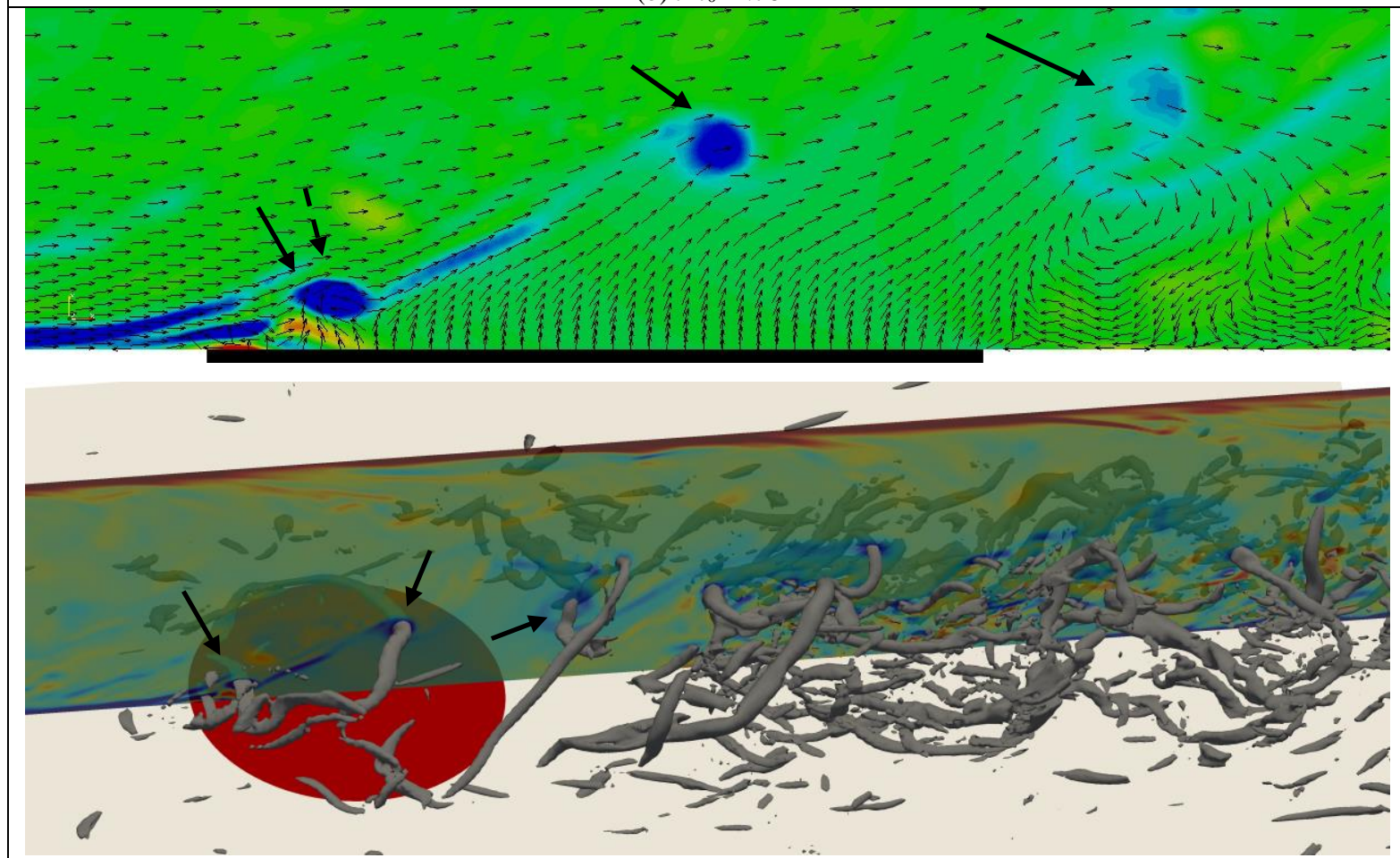

(c) $t=t_{0}+3.24$

Figure 14. Spanwise vorticity with surface vector at three different time: (a) $t=t_{0}+2.40$; (b) $t=t_{0}+2.76$, and (c) $t=t_{0}+3.24$. The black bar shows the jet-exit location $(-1<x / H<1)$. The dashed arrows indicate the process of a shedding of crossflow boundary layer vortex. The solid arrows present different phases of a fallen-off vortex. Figure (c) shows the iso-surface of $Q=15$ at time $t_{0}+3.24$. 